\title{
Vehicle Attitude Estimation in Adverse Weather Conditions using a Camera, a GPS and a 3D road Map
}

\author{
Rachid Belaroussi, Jean-Philippe Tarel and Nicolas Hautière
}

\begin{abstract}
We investigate the scenario of a vehicle equipped with a camera and a GPS driving on a road whose 3D map is known. We focus on the case of a road under fog or/and snow conditions. The GPS is used to estimate the vehicle pose and yaw and then the 3D road map is projected onto the camera image. The vehicle pitch and roll angles are then refined by fitting the projected road to detected road markings. Finally, we discuss the pros and cons of the obtained road registrations in the images and of the vehicle pitch-roll estimates, with respect to the vehicle dynamics and the driving environment, in adverse weather conditions.
\end{abstract}

\section{INTRODUCTION}

Advance Driver Assistance Systems (ADAS) are designed to enhance safety and traffic flow. The most widely used type of ADAS is in-vehicle navigation system: it typically uses a GPS receiver and a digital map to indicate the location and path of the vehicle. However the GPS only delivers the vehicle position. The vehicle pitch can be estimated using a two antenna GPS at the vehicle front and rear [1]. With the same approach, the complete estimation of the vehicle altitude requires a three antenna GPS.

Complementary navigation sensors such as odometers, accelerometers and gyroscopes, are commonly used to perform the dead reckoning task. They constitute the inertial measurement unit IMU [2] and provide first or secondorder derivative of the position and attitude (yaw, pitch and roll) of the vehicle. Except for the inertial sensors used in missile, aircraft or submarines, which have high cost, size and power consumption, the IMU sensors (MEMS based) are not accurate and they are sensitive to car vibrations. The downward and sideway velocity components cannot be neglected and cause biases on the pitch and roll angles that can be filtered with a vehicle model, as used in Inertial Navigation System (INS). This kind of system is sensitive to motion model uncertainties. Moreover, inertial sensors cannot be used for trajectory forecasting at long distance or when the road shape varies rapidly (turns, road bumps ...). In our approach, no information about the vehicle model and no assumption on the vehicle motion are used.

Detecting the road ahead of a vehicle is crucial to assess the degree of conformity of information regarding the position and velocity provided by the navigation system. Road following systems use active (laser and radar) or passive (camera) sensors [3]. Laser and radar are useful in rural areas to find road boundaries but fail on multi-lane roads. Another issue is that the radar energy can be reflected by objects

Authors are with the University Paris Est, IFSTTAR, LEPSiS, $58 \mathrm{Bd}$ Lefebvre, 75015 Paris, France that can be overridden safely like a metal plate or a Botts' dot on the road. To solve this difficulty, [4] combines the structure map extracted from a single plane scanning radar with an occupancy map deduced from a monocular camera data (feature detection and tracking), to estimate the road boundaries. When the road has no other physical bound than white stripes this approach is not relevant.

A monocular camera pointing at the scene in front of the vehicle is a very informative source. Algorithms developed to estimate the lane position usually detect lane features to which a road model is fitted. The road model can be a polyline or a parametric function such as a polynomial approximation, see for instance [5], [6], [7]. However, the vehicle attitude cannot be obtained without ambiguity using a single camera. Knowledge on the road are required, for instance the road width. With two cameras, stereovision can be used to estimate the road shape as well as the vehicle attitude [8], [9]. This kind of approach, relying on cameras, is subject to the occlusion problem: it could be greatly improved by the a priori knowledge of the map of the road viewed by the camera, typically with a map and a GPS.

More recent approaches assume the road network has been surveyed accurately beforehand. In [10] a digital map of the road is merged with data from a RTK-GPS, an odometer and a gyrometer to extract the modeling in clothoids of the road. The map is used as a geometrical constraint in the egopositioning of the vehicle and for map-matching purposes. In [11] a loosely coupled GPS/INS system is used with a camera to estimate an accurate vehicle localization, its lateral and longitudinal position and its yaw. The map is made of polylines and area information divided into three classes (line landmark, road surface and background). This approach can estimate the vehicle localization and its yaw, even if the road boundaries are not precisely localized, but not its pitch and roll.

In [12] a numerical map, a GPS and a color camera are used to detect the road boundaries. The road skeleton is modeled as junctions of connected piecewise continuous lines. The road model is made of a drivable area and two road sides. Consecutive positions of the vehicle are used to estimate the vehicle orientation and position in the current frame. Knowing the vehicle yaw, the road map is projected onto the image. The road borders detection is refined using a color model of the road image and the road width extracted from the map attributes. The initial stage of road map projection in the image is the same as the one proposed in the present paper. The improvement over the second stage is that it uses lane markings in gray scale images and explicitly 
incorporates the road bank and pitch (and thus does not assume a planar movement of the vehicle) and is able to estimate vehicle roll and pitch.

The remainder of the paper is organized as follows. Section II presents the digital 3D road map characteristics, the vehicle apparatus used and how the map points are projected onto the image plane. Section III describes the estimation of the vehicle yaw from the GPS data, and of the road bank and slope from the 3D road map, knowing the current position of the vehicle from the GPS. The process of map registration in the image by way of a distance transform to the extracted lane marking elements is explained in section IV. An estimation of the vehicle pitch and roll angles is deduced from this registration. In section $\mathrm{V}$, thanks to the introduction of an image ground-truth, the accuracy of the road lanes registration in the image is evaluated by a mean distance to the reference. The accuracy of the results is evaluated on real data from a test track, under adverse weather conditions.

\section{EUCLIDEAN TRANSFORMATION FROM WORLD TO CAMERA FRAME}

\section{A. Digital Map Description}

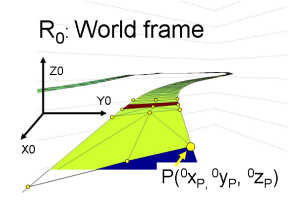

Road marking georeferences

(a)

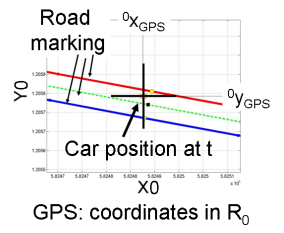

(b)

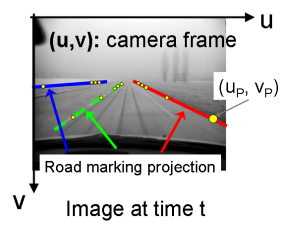

(c)
Fig. 1. Knowing the 3D topography of the road (a), the GPS coordinates of the vehicle (b) and the camera calibration, the pan-tilt-roll angles of the camera are estimated and the 3D points of the road map are projected in the image (c).

The Satory test road (France) is a $3.5 \mathrm{~km}$ track the 3D topography of which was accurately measured by professional land surveyors. Points along the road were accurately georeferenced: along each section of the 2-lane road a triplet of points is measured, one point for each of the three lines drawn on the road, as shown in Fig. 1(a). The red and the blue lines define the borders of the road, while the green line shows the middle of the road, see Fig. 1(b).

The map is made of 380 triplets of 3D points which makes a total of $N_{\text {Map }}=1140$ points. This set is named $\mathcal{S}_{M a p}$ in the following. A feature point $P_{k} \in \mathbb{R}^{3}$ of $\mathcal{S}_{\text {Map }}$ is indexed by an integer $k \in\left[1, N_{M a p}\right]$.

\section{B. In-Vehicle Apparatus}

The experimental setup includes a Real Time Kinematic (RTK) GPS system and a camera, both mounted on a Peugeot 307 car. The camera framerate is $25 \mathrm{~Hz}$ while the GPS frequency is $\approx 20 \mathrm{~Hz}$ : data are timestamped in order to be associated. The GPS is slower than the image grabber: a lag in the data recording process induces a positioning imprecision growing with the vehicle speed. Therefore, the estimation of the vehicle yaw needs some data filtering: this stage is explained thereafter in this paper. The camera provides quarter PAL $384 \times 288$ grayscale images. The relative position between the GPS and the camera were accurately measured.

GPS data are Cartesian coordinates expressed in the same world frame $\Re_{0}$ as the $3 \mathrm{D}$ road map points.

\section{Pinhole Camera}

In order to project a point $P$ of the real world onto the image plane, three reference frames are introduced: the world frame is denoted $\Re_{0}$, the GPS frame $\Re_{g p s}$, and the camera frame $\Re_{\text {cam }}$. The image plane is referenced by two coordinates $(u, v)$ where $(0,0)$ is the top left corner of the image. The camera center $O_{c a m}$ projection in the image plane is denoted $\left(u_{0}, v_{0}\right)$. In the case of a pinhole perspective camera model, the projection of $P$ in the image plane is the point ${ }^{\mathbb{I}} P=\left(u_{P}, v_{P}\right)$ with the following coordinates:

$$
{ }^{\mathbb{I}} P=\left(u_{P}, v_{P}\right) \quad \text { with }\left\{\begin{array}{l}
u_{P}=u_{0}-\lambda_{x} \frac{{ }^{c a m} y_{P}}{\operatorname{cam}^{c} x_{P}} \\
v_{P}=v_{0}-\lambda_{y} \frac{{ }^{c a m} z_{P}}{\operatorname{cam}_{P}}
\end{array}\right.
$$

where $f, \lambda_{x}=f / \Delta_{x}$ and $\lambda_{y}=f / \Delta_{y}$ are the camera intrinsic parameters, and coordinates of $P$ in the camera frame are ${ }^{\text {cam }} P=\left({ }^{\text {cam }} x_{P},{ }^{\text {cam }} y_{P},{ }^{\text {cam }} z_{P}\right)$.

The camera has no pan nor roll and a fixed tilt $\theta_{0}^{\text {cam }}$ with regard to the vehicle axis: it is the angle between the $X_{g p s}$ and $X_{c a m}$ axes (rotation about the $Y_{\text {cam }}$ axis). The transformation between $\Re_{\text {gps }}$ and $\Re_{\text {cam }}$ frames also includes the translation from $O_{g p s}$ to $O_{c a m}$ which is estimated with a centimeter accuracy.

The transformation between the GPS frame and the world frame involves three angles: $\psi$ for yaw, $\theta$ for pitch, $\phi$ for roll, and a translation vector. The vehicle position is expressed in $\Re_{0}$ as: $O_{g p s}=\left({ }^{0} x_{g p s},{ }^{0} y_{g p s},{ }^{0} z_{g p s}\right)$. The roll is the sum of the road bank $\phi_{0}$ and of the vehicle roll $\phi_{V}$, the pitch is the sum of the road slope $\theta_{0}$ and of the vehicle pitch $\theta_{V}$.

\section{ESTIMATES FROM THE GPS AND THE 3D MAP}

\section{A. Estimation of the Vehicle Yaw}

The yaw $\psi$ is estimated at current time $t_{0}$ using the vehicle trajectory. As mentioned in [13], the imprecision of the yaw estimated using a first order derivative (i.e. $\left.O_{g p s}\left(t_{0}\right)-O_{g p s}\left(t_{0}-1\right)\right)$ of the GPS data can lead to an imprecision of 1 radian. To avoid this level of inaccuracy, the vehicle yaw is filtered over $N_{0}$ previous GPS points.

Let $\mathbb{F}_{t_{0}}$ be the set of the $N_{0}=15 \mathrm{GPS}$ points prior to time $t_{0}$ after projection on the horizontal plane of $\Re_{0}$. To filter out the GPS noise, a second degree polynomial fitting is performed on the subset $\mathbb{F}_{t_{0}}$.

Let us denote $\left(\overline{{ }^{0} x}, \overline{{ }^{0} y}\right)$ the coordinates of the mean point of $\mathbb{F}_{t_{0}}$. Denoting the best polynomial fit as $y(x)-\overline{{ }^{0} y}=$ $a\left(x-\overline{{ }^{0} x}\right)^{2}+b\left(x-\overline{{ }^{0} x}\right)+c$, then the yaw angle at time $t_{0}$ is defined as:

$$
\psi=\tan ^{-1}\left(2 a\left({ }^{0} x_{g p s}\left(t_{0}\right)-\overline{{ }^{0} x}\right)+b\right)
$$



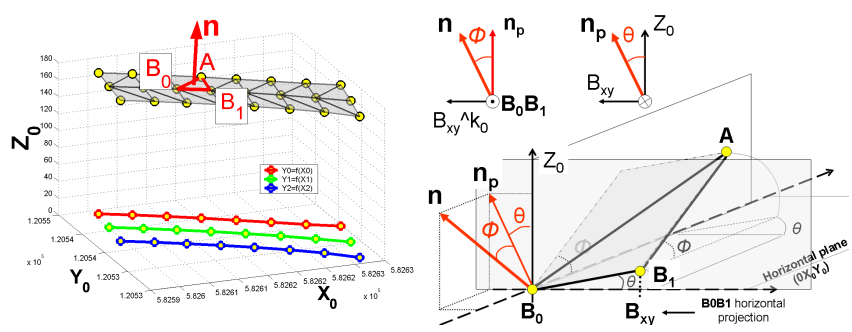

Fig. 2. The 3D map is made of bands, each band is made of 6 vertices (yellow circles) and 4 triangular faces. The road slope and bank angles are computed from the normal vector $\mathbf{n}$.

\section{B. Estimation of the Road Slope and Bank}

Both angles are calculated using the 3D road map. The sampled points are drawn as yellow circles in Fig. 2. Let us assume the vehicle GPS position is over a triangle $A B_{0} B_{1}$ where $B_{0} B_{1}$ are two adjacent points of the same line of the road. One can compute the normal vector of the $A B_{0} B_{1}$ triangle as the cross product $\mathbf{n}=\mathbf{B}_{0} \mathbf{B}_{1} \wedge \mathbf{B}_{0} \mathbf{A}$. Let us call $\mathbf{B}_{\mathbf{x y}}$ the orthogonal projection of $\mathbf{B}_{\mathbf{0}} \mathbf{B}_{\mathbf{1}}$ on the horizontal plane of $\Re_{0}$ :

$$
\mathbf{B}_{\mathbf{x y}}=\left(\begin{array}{lll}
{ }^{0} x_{B_{1}}-{ }^{0} x_{B_{0}}, & { }^{0} y_{B_{1}}-{ }^{0} y_{B_{0}}, \quad 0
\end{array}\right)^{t}
$$

and let $\mathbf{n}_{\mathbf{p}}$ be the orthogonal projection of $\mathbf{n}$ on the $\left(Z_{0}, \mathbf{B}_{\mathbf{x y}}\right)$ vertical plane containing the $B_{0} B_{1}$ segment. We set $\mathbf{k}_{0}$ as the unitary vector along $Z_{0}$ axis. As shown in Fig. 2 right, $\phi$ and $\psi$ angles are the oriented angles $\angle\left(\mathbf{n}_{\mathbf{p}}, \mathbf{n}\right)$ and $\angle\left(\mathbf{k}_{\mathbf{0}}, \mathbf{n}_{\mathbf{p}}\right)$ respectively. Therefore, one can compute the road bank angle in $\Re_{0}$ as:

$$
\phi_{0}=\sin ^{-1}\left(\frac{\mathbf{n} \cdot\left(\mathbf{B}_{\mathbf{x y}} \wedge \mathbf{k}_{\mathbf{0}}\right)}{\|\mathbf{n}\|\left\|\mathbf{B}_{\mathbf{x y}}\right\|}\right)
$$

and the road slope as:

$$
\theta_{0}=-\tan ^{-1}\left(\frac{\mathbf{n} \cdot\left(\mathbf{B}_{\mathbf{x y}} /\left\|\mathbf{B}_{\mathbf{x y}}\right\|\right)}{\mathbf{n} \cdot \mathbf{k}_{\mathbf{0}}}\right)
$$

\section{RoAd MAP REgistration IN THE IMAGE}

\section{A. Lane Marking Elements Extraction}

The best extraction algorithm of lane marking elements from [14] is applied on the image, followed by a skeletonization to obtain lane markings centers. Fig. 3(b) shows the detected pixels (black pixels) from the image in Fig. 3(a). The road map data is projected in the image with the road slope $\theta_{0}$ and bank angle $\phi_{0}$ estimated at the current position $O_{g p s}$ and with the estimated vehicle yaw $\psi$. This projection is drawn in dotted line in Fig. 3(b): one can notice the gap between the projected map and the reference lanes positions. To evaluate the additional vehicle pitch $\theta_{V}$ and roll $\phi_{V}$, the $3 \mathrm{D}$ road map is projected on the image and registered on the extracted lane marking elements.

\section{B. Distance Transform}

The distance transform [15] to the set of lane markings centers is computed, as shown in Fig. 3(c). The used distance in the distance transform is the $L_{1}$ or city block distance. The result of the distance transform is stored in an image denoted $D T$ hereafter. The top and bottom of the image are removed so as to discard the vehicle and the top of the sky.

\section{Optimization Criterion}

A Chamfer matching is done using the $D T$ image as a potential energy to be minimized, see Fig. 3(d). The coordinates of the projection in the image of the points of $\mathcal{S}_{\text {Map }}$ depends on the Euler angles $(\theta, \phi, \psi)$. Let us consider a line segment $\left[\begin{array}{ll}P_{k} & P_{k+1}\end{array}\right]$ of the 3D map. Its projection in the image is the line segment $\left[{ }^{\mathbb{I}} P_{k} \quad{ }^{\mathbb{I}} P_{k+1}\right]$. Points along all visible line segments are sampled. This set is denoted ${ }^{\mathbb{I}} \mathcal{L}(\theta, \phi, \psi)$ :

$$
{ }^{\mathbb{I}} \mathcal{L}(\theta, \phi, \psi)=\left\{{ }^{\mathbb{I}} M \in\left[{ }^{\mathbb{I}} P_{k}{ }^{\mathbb{I}} P_{k+1}\right], P_{k}, P_{k+1} \in \mathcal{S}_{M a p}\right\}
$$

The Chamfer matching consists in finding the minimal mean distance between the projected lanes and the lane centers extracted in the image. The score function is thus defined as follow:

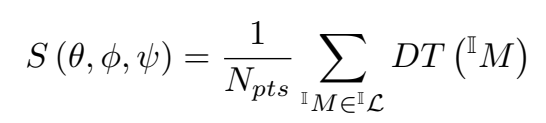

where $N_{p t s}=\operatorname{Card}\left({ }^{\mathbb{I}} \mathcal{L}(\theta, \phi, \psi)\right)$.

At current time $t_{0}$, the vehicle yaw $\psi$ is estimated as explained previously. To simplify the presentation, we here consider that the estimated yaw is valid and does not need any refinement. This assumption is questionable but may be easily relaxed. Under this assumption, the score $S$ reduces to a function of $\theta$ and $\phi$. We recall that the road slope and bank angle are respectively $\theta_{0}$ and $\phi_{0}$. The calculation of $S(\theta, \phi)$ is performed in a neighborhood of these two values:

$$
\begin{gathered}
\theta \in\left[\theta_{0}-\alpha_{\max }, \theta_{0}+\alpha_{\max }\right] \\
\phi \in\left[\phi_{0}-\alpha_{\max }, \phi_{0}+\alpha_{\max }\right]
\end{gathered}
$$

with $\alpha_{\max }=6^{\circ}$ in our experiments.

\section{Estimation of the Vehicle Roll and Pitch}

Fig. 3(e) shows the values of $S(\theta, \phi)$ around $\left(\theta_{0}, \phi_{0}\right)$ where warm colors correspond to high values and cold colors to small values. The vehicle pitch $\theta_{V}$ and roll $\phi_{V}$ at time $t_{0}$ are then deduced from the location of minimum of $S$ after discretizing roll and pitch:

$$
\left(\theta_{V}, \phi_{V}\right)=\arg \min _{\theta, \phi} S\left(\theta_{0}+\theta, \phi_{0}+\phi, \psi\right)
$$

The uncertainty about these two estimates can be obtained by the quadratic modeling of $S$ in the neighborhood of the minimum location.

For example, at point $\mathrm{A}$ of the test track of Fig. 5, the slope and bank angles are $\left(\theta_{0}, \phi_{0}\right)=\left(-1.7^{\circ},-2.9^{\circ}\right)$. At point A, Fig. 3(d) plots on the $D T$ image the projected lanes ${ }^{\mathbb{I}} \mathcal{L}\left(\theta_{0}, \phi_{0}\right)$ in dotted lines. The mean distance tranform value is $\overline{L_{1}}=18.1$ pixels. The resulting lanes ${ }^{\mathbb{I}} \mathcal{L}\left(\theta_{0}+\theta_{V}, \phi_{0}+\phi_{V}\right)$ after minimization of $\mathbf{S}$ are at a mean distance of $\overline{L_{1}}=6.1$ pixels to the extracted road marking elements. The set ${ }^{\mathbb{I}} \mathcal{L}\left(\theta_{0}+\theta_{V}, \phi_{0}+\phi_{V}\right)$ is plotted as solid lines on Fig. 3(d). The minimum is achieved for $\left(\theta^{*}, \phi^{*}\right)=$ $\left(1.9^{\circ},-5.9^{\circ}\right)$ so that $\left(\theta_{V}, \phi_{V}\right)=\left(3.6^{\circ},-3^{\circ}\right)$. The vehicle 


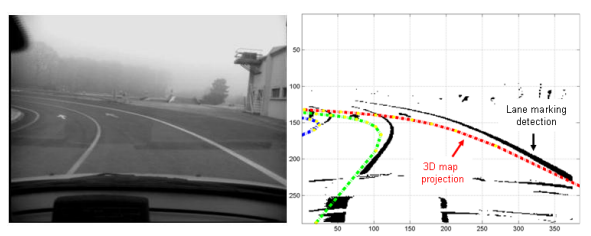

(a)

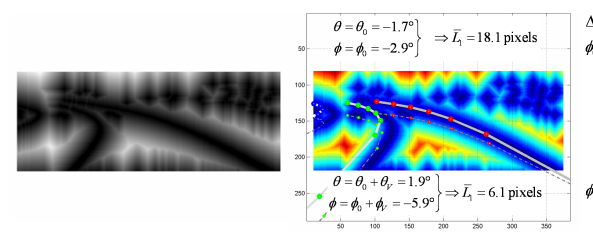

(c)

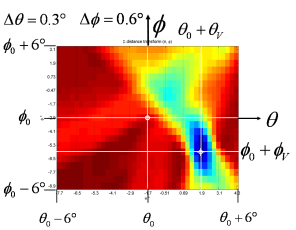

(e)

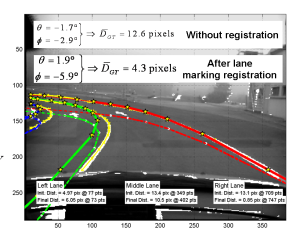

(f)

Fig. 3. (a) Source image. (b) Lane marking detection in black, projected road map in the image (dotted lines) knowing the GPS position of the car. (c) The distance transform displays the distance from each pixel to the closest marking pixel. (d) Distance transform shown using a colormap which is decreasing from red to blue. The gray lines are the road lanes registered after minimization of the mean $\overline{L_{1}}$ distance. (e) The mean $\overline{L_{1}}$ distance as a function of pitch $\theta$ and roll $\phi$ angles. The location of its minimum is used to estimate the vehicle pitch and roll. (f) Dashed lines are the initial road lanes projection, the solid lines are the results after lanes registration.

pitch and roll are particularly high at this point $\mathrm{A}$ of the circuit, due to entering into a hairpin turn with high speed.

Fig. 3(f) shows the result of the road lanes registration on the source image at point A. The initial location of the lanes is plotted in dotted line. The final location obtained after optimization of $\mathrm{S}$ is represented by the solid lines. The ground-truth is plotted as yellow segments. The mean distance $\bar{D}_{G T}$ between the projected lanes ${ }^{\mathbb{I}} \mathcal{L}$ and the groundtruth is initially $\bar{D}_{G T}\left(\theta_{0}, \phi_{0}\right)=12.6$ pixels and is reduced to $\bar{D}_{G T}\left(\theta^{*}, \phi^{*}\right)=4.3$ pixels after $S$ minimization.

\section{EXPERIMENTAL RESULTS}

The GPS and camera data were acquired on a portion of the Satory test track, as shown in Fig. 5, and contains various and typical situations such as straight portions, sharp turns or S-shape bend, and road bumps. A few interesting points are used to illustrate the obtained results, they are labeled by letters A to $\mathrm{K}$.

The images were acquired in adverse weather conditions with fog and snow: road marks are heavily occluded, with small contrasts and in presence of many false positives due to the tracks left by previous vehicles. Fig. 4 shows some examples of the initial and final 3D projected map in the image, with the ground-truth on the interesting points. The dotted lines show the initial lanes projection (mean distance $D_{G T}^{0}$ to ground-truth). The lanes after registration in the image are plotted as solid lines (distance $D_{G T}^{*}$ to groundtruth). The thin yellow lines represent the manually labeled ground-truth.

\section{A. Distance to Ground-truth in the Image}

To evaluate the accuracy of the road map registration in the image, we manually built a ground-truth on sampled images. On a sequence of 1920 images, one image every 10 frames is picked and the location of the three lanes present in the images are hand labeled. For each of these images, a set of ground-truth segments ${ }^{\mathbb{I}} \mathcal{L}_{G T}$ is thus available. An example of ground-truth is drawn as yellow lines in Fig. 3(f).

When available, the mean distance between the groundtruth and the projected road map is calculated. This mean distance is computed as the Modified Hausdorff Distance (MHD) [16], i.e as the average of $d\left({ }^{\mathbb{I}} M,{ }^{\mathbb{I}} \mathcal{L}_{G T}\right)$ over the points ${ }^{\mathbb{I}} M \in{ }^{\mathbb{I}} \mathcal{L}_{G T}(\theta, \phi, \psi)$ of the projected map and the reference location of the lanes:

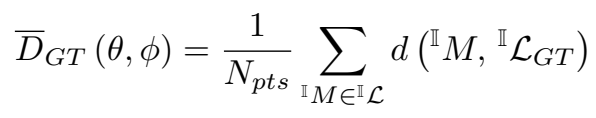

Fig. 6 plots $\bar{D}_{G T}$ versus the frame number over the test sequence. We computed the statistics of $\bar{D}_{G T}^{0}=\bar{D}_{G T}\left(\theta_{0}, \phi_{0}\right)$ and $\bar{D}_{G T}^{*}=\bar{D}_{G T}\left(\theta^{*}, \phi^{*}\right)$ (before and after registration) over the test images. Initially, the average $\bar{D}_{G T}^{0}$ over the sequence is 5.6 pixels with a standard deviation of 2.95 pixels. After registration, the average $\bar{D}_{G T}^{*}$ over the sequence is 1.9 pixels with a standard deviation of 1.0 pixel. As a conclusion, the road lanes registration improves the accuracy of lanes localization in the image.

\section{B. Vehicle Pitch and Roll}

Knowing the road bank and pitch angles, our algorithm estimates the vehicle pitch $\theta_{V}$ and roll $\phi_{V}$ angles. The optimal angles range is $\left[-6^{\circ},+6^{\circ}\right]$ and the accuracy is $\Delta \theta=0.03^{\circ}$ and $\Delta \phi=0.06^{\circ}$. It is compared to the reference angles $\theta_{V}^{G T}$ and $\phi_{V}^{G T}$ computed from the image based ground-truth. No gyrometer measurements were available for this test data and, as mentioned in the introduction, a not-too-expensive MEMS-based IMU is too noisy to be used.

Fig 7(a) plots the vehicle pitch angle $\theta_{V}$ versus the frame number of the test sequence as a continuous red curve. The ground-truth $\theta_{V}^{G T}$ is plotted as a black dotted curve. One can see that the two curves significantly overlap: most of the time, the estimated vehicle pitch equals the reference one. In $83 \%$ of the cases the deviation $\left|\theta_{V}-\theta_{V}^{G T}\right|$ is less than $0.6^{\circ}$, and for $89 \%$ of the images $\left|\theta_{V}-\theta_{V}^{G T}\right| \leq 1^{\circ}$. The error $\theta_{V}-\theta_{V}^{G T}$ seems normally distributed over the sequence with a mean of $\overline{\theta_{V}-\theta_{V}^{G T}}=0.1^{\circ}$ and with a standard deviation of $0.5^{\circ}$.

Fig 7(b) displays the estimated vehicle roll $\phi_{V}$ versus the frame number as a continuous red curve. The reference vehicle roll angle $\phi_{V}^{G T}$ is plotted as a black dotted curve. The estimation of the roll angle is less accurate than it is for the pitch. The outliers are due to lack of information about the lane markings in the image due to snow on the road. However, for $81 \%$ of the images the deviation $\left|\phi_{V}-\phi_{V}^{G T}\right|$ is less than $1^{\circ}$. The mean error along the test sequence is $\overline{\phi_{V}-\phi_{V}^{G T}}=0.4^{\circ}$ with a standard deviation of $1.1^{\circ}$.

The kind of statistics on the roll and pitch errors, here reported, are rarely given in the literature on real data. 


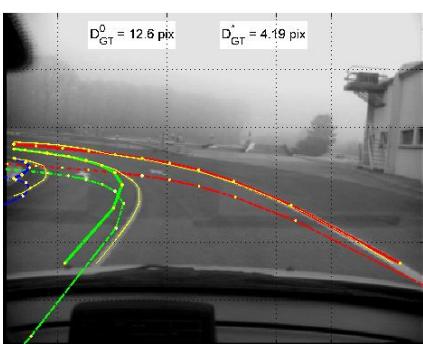

(A)

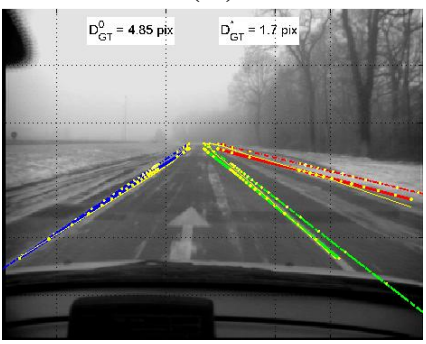

(C)

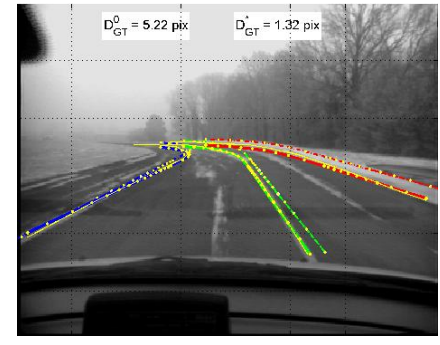

(E)

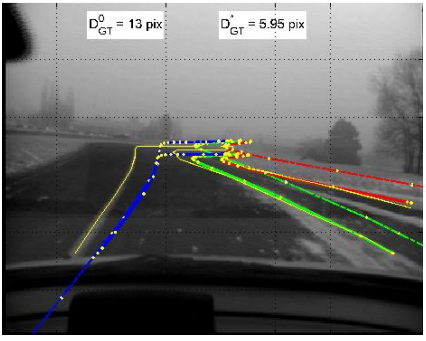

(G)

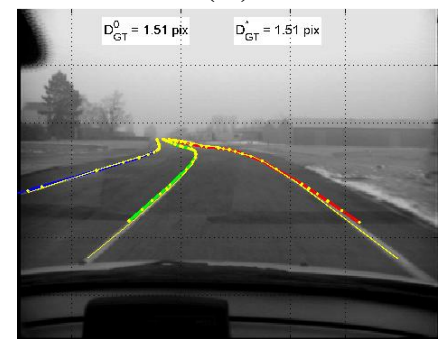

(I)

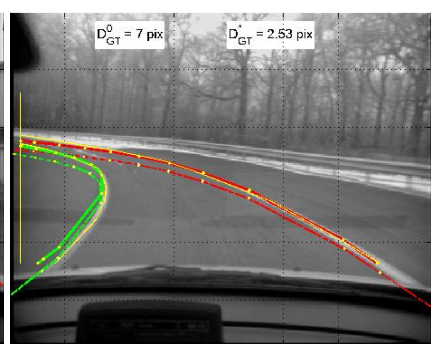

(B)

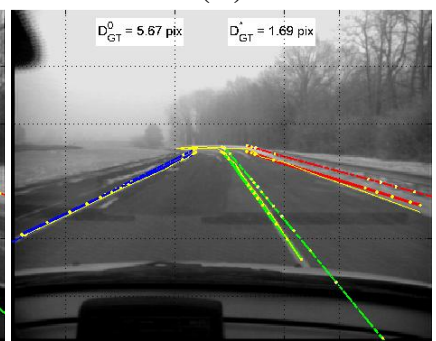

(D)

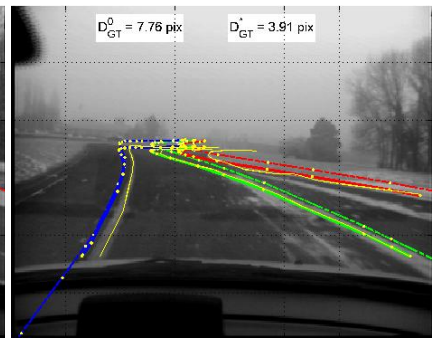

(F)

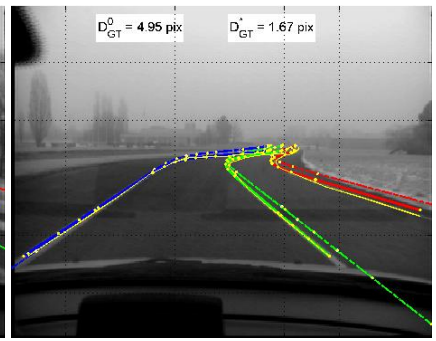

$(\mathrm{H})$

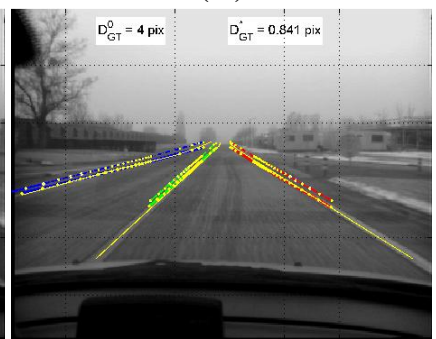

(J)
Fig. 4. Road markings projection (dotted lines) and registration (solid lines) in images of points A to $\mathrm{J}$ of the Satory track.

This illustrates the need for common reference databases in Intelligent Vehicle community.

\section{Sensitivity to Perturbations}

In our experiments, high quality data are used (3D map of the test track, RTK-GPS). We still have to evaluate the behavior and performance of the approach regarding the

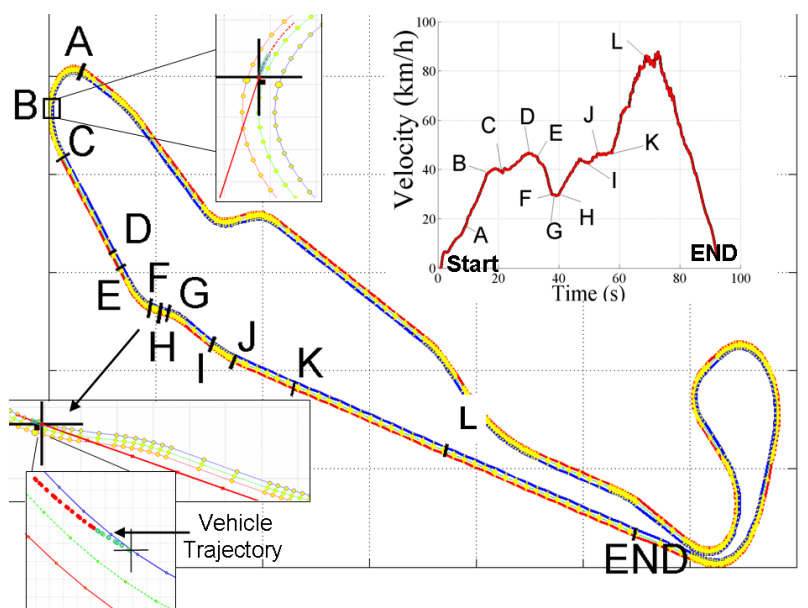

Fig. 5. Velocity profile on a circuit portion from point A to the end point. Sections close to point $\mathrm{B}$ and $\mathrm{F}$ are zoomed in with the vehicle trajectory drawn as dots and the vehicle yaw as a red line.

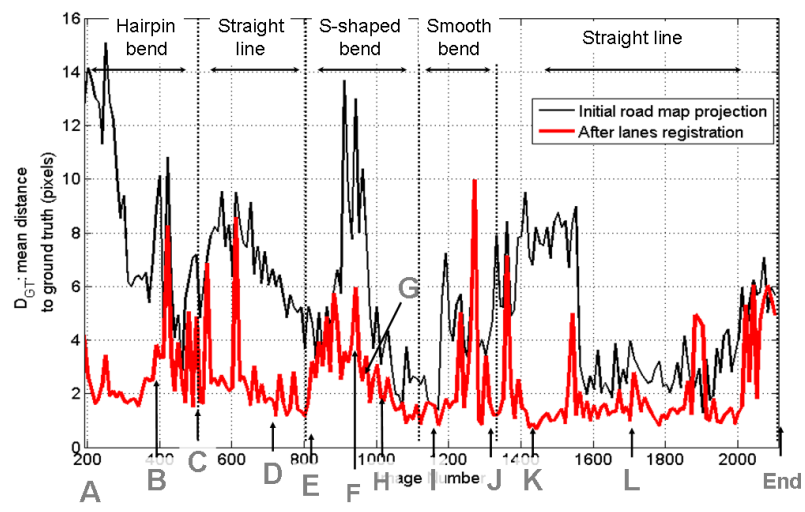

Fig. 6. Mean distance $\bar{D}_{G T}$ between projected road map and ground-truth versus the image number (time stamp). The mean distance $\bar{D}_{G T}\left(\theta_{0}, \phi_{0}\right)$ of the road lanes from the GPS is plotted in black. After registration on the image(red plot), the mean distance is smaller most of the time.

following perturbations:

- uncertainty of the positioning system,

- uncertainty and spatial sampling of the 3D points of the road markings.

Our approach does not provide an improvement in the positioning, the vehicle position being directly obtained from the GPS. We experienced that the RTK-GPS accuracy is sufficient. We observed that an error in the vehicle yaw estimation induces important errors on lanes registration. This happens particularly when the vehicle slides, especially when the car rotates around a vertical axis without changing the direction of its trajectory, as in Fig. 4(G). The yaw angle can be refined similarly to the pitch and roll angles.

On the linear portions of the track, the points in the digital map are sampled every 20 meters. The longitudinal sample frequency is increased up to one triplet every four meters on curved sections of the road. In our investigations, we observed that such a high spatial sampling rate is required only on curved sections. The total road width is 7 meters on average, and the accuracy of surveyed points is important 


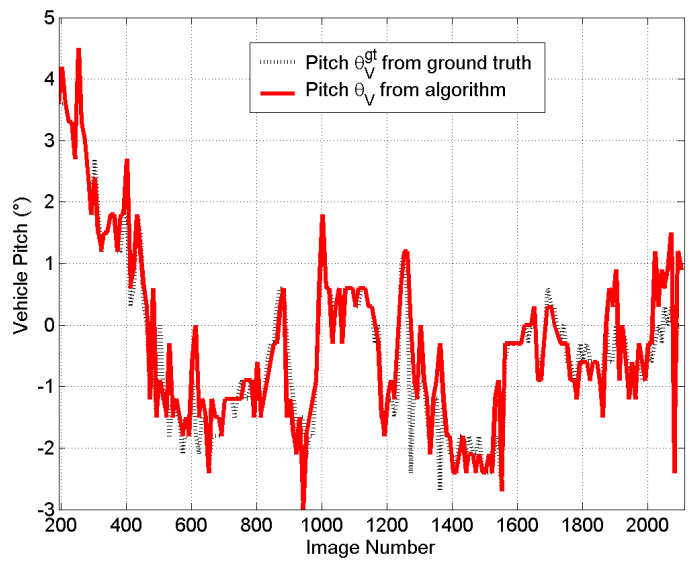

(a)

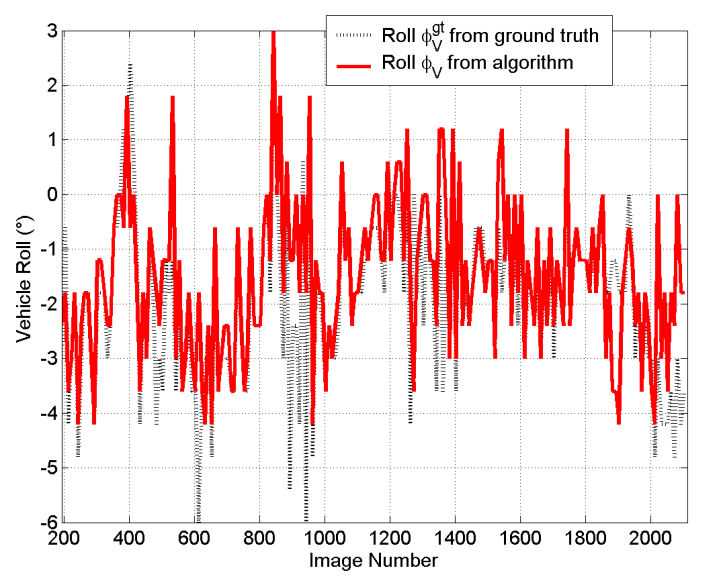

(b)

Fig. 7. (a) Vehicle pitch angle (in ${ }^{\circ}$ ) evolution over time: estimation $\theta_{V}$ (red) and reference pitch $\theta_{V}^{G T}$ (dotted black line). (b) Vehicle roll angle ( ${ }^{\circ}$ ) versus time: estimation $\phi_{V}$ (red) and reference roll angle $\phi_{V}^{G T}$ (black).

in the horizontal plane. The accuracy of their altitude is not relevant because the registration mainly takes into account the near field of the camera. A locally constant altitude could be inferred and set to ${ }^{0} z_{\text {gps }}-H_{0}$ where $H_{0}$ is the initial vertical distance of the GPS from the ground.

\section{CONCLUSIONS AND FUTURE WORKS}

Using a digital road map made of triplets of 3D points along markings and knowing the RTK-GPS position of the vehicle, we computed the road bank and slope angles, and estimated the vehicle yaw. When using these estimates with the camera calibration, the projected map in the image is 5.6 pixels in average from the reference on our test sequence.

Then we proposed a vehicle attitude estimation algorithm which precisely localizes the lane markings in images and estimates the vehicle pitch and roll without using any inertial sensors. Using the lane marking elements extracted in the image, we proposed to use the Chamfer matching approach to refine the road marks localization. After this registration process, the road lanes are localized with an average error of 1.9 pixels to the reference location in the image. The vehicle pitch and roll are also estimated by our algorithm with an error lower than $1.0^{\circ}$ in most of the cases $(90 \%)$. We tested the proposed algorithm in adverse weather conditions with fog and snow, and with the vehicle in various dynamical states.

Future work consists in reducing the processing time in order to incorporate our system into a prototype and then assessing the behavior of the algorithm in real time conditions. The refinement of the vehicle yaw angle is also interesting to consider by following the same approach. Potential applications include all aspects of ADAS, especially safety in adverse weather conditions, visual guidance by augmented reality. Other nomad applications using devices such as smart phones (that have built-in camera and GPS/INS sensors) can be envisioned also for both pedestrian and biker guidance.

\section{REFERENCES}

[1] H. Bae, J. Ryu, and J. Gerdes, "Road grade and vehicle parameter estimation for longitudinal control using gps," in Proceedings of IEEE Conference on Intelligent Transportation Systems (ITSC'01), 2001, pp. 166-171.

[2] I. Skog and P. Handel, "In-car positioning and navigation technologies - a survey," IEEE Transactions on Intelligent Transportation Systems, vol. 10, no. 1, pp. 4-21, 2009.

[3] J. McCall and M. Trivedi, "Video based lane estimation and tracking for driver assistance: Survey, system, and evaluation," IEEE Transactions on Intelligent Transportation Systems, vol. 7, no. 1, pp. 20-37, 2006.

[4] M. Darms, M. Komar, and S. Lueke, "Map based road boundary estimation," in IEEE Intelligent Vehicles Symposium (IV'10), 2010, pp. 609-614.

[5] J. Goldbeck, B. Huertgen, S. Ernst, and L. Kelch, "Lane following combining vision and dgps," Image and Vision Computing, vol. 18, pp. 425-433, 2000.

[6] J. Park, J. Lee, and K. Jhang, "A lane-curve detection based on an lcf," Pattern Recognition Letters, vol. 24, pp. 2301-2313, 2003.

[7] J.-P. Tarel, P. Charbonnier, and S.-S. Ieng, "A revisited half-quadratic approach for simultaneous robust fitting of multiple curves," in Computer vision and Computer Graphics, revised selected papers of visigrapp'07, CCIS 21, 2009, pp. 121-133.

[8] R. Labayrade and D. Aubert, "A single framework for vehicle roll, pitch, yaw estimation and obstacles detection by stereovision," in IEEE Intelligent Vehicles Symposium (IV'03), 2003, pp. 31-36.

[9] J.-P. Tarel, S.-S. Ieng, and P. Charbonnier, "Accurate and robust image alignment for road profil reconstruction," in Proceedings of IEEE International Conference on Image Processing (ICIP'07), vol. V, San Antonio, Texas, USA, 2007, pp. 365-368.

[10] R. Toledo-Moreo, D. Betaille, F. Peyret, and J. Laneurit, "Fusing gnss, dead-reckoning, and enhanced maps for road vehicle lane-level navigation," IEEE Journal of Selected Topics in Signal Processing, vol. 3, no. 5, pp. 798-809, 2009.

[11] N. Mattern, R. Schubert, and G. Wanielik, "High-accurate vehicle localization using digital maps and coherency images," in IEEE Intelligent Vehicles Symposium (IV'10), 2010, pp. 462-469.

[12] J. Alvarez, F. Lumbreras, T. Gevers, and A. Lopez, "Geographic information for vision-based road detection," in IEEE Intelligent Vehicles Symposium (IV'10), 2010, pp. 621-626.

[13] R. Gallen and S. Glaser, "Vision based tangent point detection algorithm, evaluation and validation," in Proc. Machine Vision Application (MVA'08), 2009, pp. 518-521.

[14] T. Veit, J.-P. Tarel, P. Nicolle, and P. Charbonnier, "Evaluation of road marking feature extraction," in Proceedings of 11th IEEE Conference on Intelligent Transportation Systems (ITSC'08), 2008, pp. 174-181.

[15] G. Borgefors, "Distance transformations in digital images," Computer Vision, Graphics, and Image Processing, vol. 34, no. 3, pp. 344-371, 1986.

[16] M. P. Dubuisson and A. K. Jain, "A modified Hausdorff distance for object matching," in Proceedings of International Conference on Pattern Recognition (ICPR'94), 1994, pp. 566-568. 